\title{
Anti-CD71/vcMMAE Probody-drug Conjugate CX-2029
}

National Cancer Institute

\section{Source}

National Cancer Institute. Anti-CD71/VCMMAE Probody-drug Conjugate CX-2029. NCI

Thesaurus. Code C153287.

A probody-drug conjugate (PDC) composed of a monoclonal antibody directed against the transferrin receptor 1 (TFR1;TRP1; CD71), linked to a proprietary masking peptide that covers the active antigen-binding site of the antibody through a protease-cleavable linker, and conjugated, via a valine-citrulline (VC) peptide linker, to the potent cytotoxic agent monomethyl auristatin $\mathrm{E}(\mathrm{MMAE})$, with potential antineoplastic activity. Upon administration of the anti-CD71/vcMMAE PDC CX-2029, the anti-CD71 moiety is unable to bind to CD71 antigen, which is highly expressed on both tumor and healthy dividing cells, until the masking peptide that is attached to the anti-CD71 probody is cleaved by tumor-associated proteases upon extravasation into the tumor microenvironment (TME). Local protease-mediated removal of the linker and masking peptide enables specific binding of the unmasked anti-CD71 moiety to CD71 expressed on tumor cells. Upon internalization and proteolytic cleavage, MMAE is released into the cytosol of CD71-expressing tumor cells, binds to tubulin, and inhibits microtubule polymerization, which induces both G2/M phase arrest and tumor cell apoptosis. CD71, a transmembrane glycoprotein, is a highly expressed protein present in a number of solid and hematologic cancers, but is also expressed on normal, healthy tissues. The peptide masking of CX-2029 minimizes binding of the anti-CD71 antibody moiety to normal, healthy cells and may minimize systemic toxicity, when compared to other anti-CD71 antibody-drug conjug ates (ADCs). Tumor-associated proteases are present in high concentrations and aberrantly activated in the TME. 